\title{
Role of partner support in psychosexual aspects of vulvar dermatoses
}

\author{
Tolu Adedipe, MRCOG, MBA, DIPM, DFSRH \\ Department of Obstetrics and Gynaecology, Women and Children's Hospital, Hull University Teaching Hospitals NHS Trust, Hull, UK
}

Women with vulvar dermatoses report a psychosexual effect due to the chronicity of symptoms and long-term treatment as the disabling nature of the condition impacts their sexual relationship. Partner role and support in the management of psychosexual impact are poorly understood and are not part of the routine care for increasing number of women with vulvar dermatoses. This paper provides an aide-mémoire for identifying patient concerns and supporting the partner. Holistic care of the affected women comprises assessment and management of the psychosexual impact of the disease on them and their partners through a dyadic or paired perspective. Limitations regarding the impact of increased virtual consultations, secondary to the recent global pandemic, and issues related to gender identity with sexual orientation are recognized.

Keywords: Vulvar diseases; Partner communication; Dermatoses; Psychosexual disorders; Sexual behaviour

\section{Introduction}

Women with vulvar dermatoses report a psychosexual effect due to the chronicity of symptoms and long-term treatment as the disabling nature of the condition impacts their sexual relationship. This paper provides an aide-mémoire for identifying patient concerns and supporting the partner. Holistic care of the affected women comprises assessment and management of the psychosexual impact of the disease on them and their partners through a dyadic perspective.

\section{Prevalence}

Reportedly, 1.7\% women visiting a private gynecology practice had vulvar lichen sclerosus; furthermore, lichen planus was detected via outpatient vulvar biopsies in $3.7 \%$ of all cases at a vulval clinic. Vulvar lichen sclerosus complex (VLSC) is a common disorder and a cause of up to $35 \%$ of patient visits to vulvar specialty clinics, suggesting a significant disease burden. The main symptoms of lichen sclerosus include pruritus (in 90\% cases), followed by dysuria, dyspareunia due to scarring, and/or pain [1-4]. This results in a significant impact on the sexual function/intimacy, subsequently leading to relationship difficulties [5]. Psoriasis, in addition to other vulvar skin conditions, contributes to vulvar discomfort and is the underlying cause in approximately $5 \%$ of all women with vulvar itching or burning [6].

Psychosexual problems can arise due to individual perceptions (real or not) of abnormality in sexual organs, with an impaired sexual/biological function, due to a disabling disease and/or its management, depending on the chronicity.

These problems, when complicated by a lack of partner support, lead to relationship breakdown, particularly in individuals with prior relationship difficulties and excessive worklife stressors, resulting in a growing epidemic of women who remain undiagnosed and continue to suffer from their

Received: 2021.06.06. Revised: 2021.08.01. Accepted: 2021.08.30. Corresponding author: Tolu Adedipe, MRCOG, MBA, DIPM, DFSRH

Department of Obstetrics and Gynaecology, Women and Children's Hospital, Hull University Teaching Hospitals NHS

Trust, Hull HU3 2JZ, UK

E-mail: busolade@gmail.com

https://orcid.org/0000-0003-3779-8864

Articles published in Obstet Gynecol Sci are open-access, distributed under the terms of the Creative Commons Attribution Non-Commercial License (http://creativecommons. org/licenses/by-nc/3.0/) which permits unrestricted non-commercial use, distribution, and reproduction in any medium, provided the original work is properly cited.

Copyright $\odot 2021$ Korean Society of Obstetrics and Gynecology 


\title{
Obstetrics \& Gynecology Science
}

\author{
Vol. 64, No. 6, 2021
}

increasingly complex condition. This is further complicated by a lack of support from the concerned physician due to poor psychosexual history-taking, limited training or clinical experience driven by factors such as trying to avoid embarrassment to the patient or partner, and change in the dynamics of a nascent therapeutic relationship. Appropriate, timely partner involvement may reduce the psychosexual burden, as evidenced in malignant vulvar disease management $[7,8]$.

\section{Psychosexual Impact}

It focuses on the following: 1) sexual body image, 2) sexual functioning, 3) relationship/intimacy dynamics, 4) comorbidities.

Psychosexual aspect encompasses the core trio of body image/sexual identity, sexual functioning, and sexual or intimate relationships [9]. Understanding the psychosexual impact of a disease on a woman helps the practitioner ask focused questions regarding partner support and engagement.

Sexual identity/image may be affected by the physical nature of a woman's condition; for example, scarring of the skin caused by the primary disease, as in VLSC, or as a perioperative outcome; pain; and profuse discharge that elicits the feeling of "being dirty and unappealing." These physical elements lead to reduced feelings of attractiveness and a reduced likelihood of being seen as a sexual being, thus turning off the "sexual-being" button.

Through transference to the nonperceptive partner, the affected woman projects reduced sexual attraction and therefore sexual desire. The resulting reduced desire of the partner for intimacy/copulation (in association with the physical impact of disease and/or other factors leading to reduced libido, arousal, or orgasm) further reinforces a negative body image.

Sexual function is best explained by the Masters and Johnson 4-stage model of the human sexual response cycle. The four stages are as follows: "excitement/arousal, plateau, orgasm, and resolution." These stages are brought about by the parasympathetic nervous system through the pelvic splanchnic nerves arising from the anterior rami of S2-S4. The vulvar skin is supplied by the dorsal nerve of the clitoris, genitofemoral nerve to the labia, perineal branch of the posterior femoral cutaneous nerve, and labial branches of the pudendal nerve. Female orgasms have been described as mostly clitoral, with the involvement of the distal vaginal roof, periurethral areas of the anterior vaginal wall, and introitus. Dermatoses affect any or all of these areas.

Brain imaging techniques emphasize the circular model, with the influence of psychological, biological, and sociocultural factors $[9,10]$. Likewise, sexual functioning, which is centered on the triad of desire, arousal, and orgasm and/ or penetration and which sometimes can be described as a nonlinear circular response cycle with overlapping phases [9], can be negatively impacted by physical limitations inflicted by a physical disease. Underlying comorbidities, psychological disease burden (increased anxiety and/or depression), prognostication, socioeconomic treatment/management burden, and reduced earning ability due to increased sickness absence have been demonstrated to have a negative impact on sexual functioning, largely libido [8].

The impact on relationship health may be covert due to a complex mix of human sexual response in the background of an intimate or relational setting underpinned by the woman's coping mechanisms such as self-objectification or interoceptive awareness [8] and partner's response amidst sociocultural factors.

Communication influences partner motivation. There is often a dyadic interaction between partners, which can be enhanced by and also exerts an effect on sexual communication. Studies on sexual pain have shown that intimacy improves when there is effective disclosure of sexual pain. This improvement is further enhanced if the receiving partner's attitude reflects empathy and validation [11-13].

The partner's response that is underpinned by the perception of disease burden and management and future implications should be explored either through direct questioning or via the patient. This is a skill that is poorly utilized by vulvar care practitioners and therefore needs to be developed in them. Understanding the partner's perspective elucidates the prevailing status, helping the physician provide advice and foster the development of self-help skills.

Medical, social, psychosocial, and sociocultural comorbidities contribute to disease burden. Co-existing common problems such as significant vulvovaginal atrophy and genitourinary symptoms of menopause, particularly in mature women, contribute to psychosexual burden. 


\section{Obstetrics \& Gynecology Science}

Tolu Adedipe. Partner support in psychosexual aspects

\section{The way forward}

A good, empathetic quick psychosexual history-taking session, after obtaining consent, is an efficient method to explore the psychosexual impact of disease on patient and her partner. This is done in stages and includes obtaining a focused medical history, past social history, and past sexual or relationship history, along with assessing the patient's and her partner's concerns and expectations given their socioeconomic outlook.

Identifying co-existent sexual disorders, as listed in Table 1, that have an impact on the woman's disease condition and partner engagement is important [14]. A history of rape, coercive control, or previous sexual harm or difficulties [14] may warrant further exploration and a specialist referral.

In addition to the benign chronic vulvar disease, other medical comorbidities that may impact the patient's symptomatology, such as poorly controlled diabetes or morbid obesity, should be identified. The same applies to the partner. Medication history is important because selective serotonin reuptake inhibitors and some antipsychotics medications are well known for their negative effect on libido.

The ideas, concerns, and expectations of the patient and partner can be explored alongside the combined readiness of the couple to optimize their sexual and relationship wellbeing. One must be aware of the "cishet norm" as the couple's ideas of sexual well-being and relationship wellness may be incongruent with physician's opinions. History-taking, which may be time-consuming, can be facilitated using miniquestionnaires, such as the Female Sexual Function Index.

Examination should be conducted to detect other conditions that may limit sexual intercourse, such as vaginal capacity and pelvic floor tonicity. Impaired sensation or oversensitivity should be identified as they are related to the impairment of the female sexual refractory period [15].

Management has to be initiated with the full consent of the women and her partner, with a readiness for subsequent referral to psychosexual practitioners, therapists, and/or sexologists. The partner also receives help through active participation in disease management by attending vulvar clinic appointments or other specialist sessions/consultations, such as those for couple therapy, sex therapy, and psychotherapy, as these provide opportunities to discuss the limitations inflicted by the disease as well as its treatment or possibility of its deterioration.

It is not unusual for vulvar lesions to be primarily identified by sexual partners before clinical presentation. This often breaks the "ice" as the "sharing whilst caring" behavior model has a positive impact on disease management and on relationship health and sexual satisfaction $[11,12]$.

Negative attitudes should be identified and corrected early. For a persistent nonengaging partner, accepting the option of nonengagement while supporting the patient may be a situation of compromise, especially if support has been declined.

Other negative attitudes include the coerciveness of a partner who either dismisses the disease condition or acts as a barrier to appropriate treatment. Early identification of such attitudes with appropriate alterations in care plans limits the impact of negative behavior and may include the provision of psychological or other civic help [14].

Toward the end of treatment, some partners are reluctant to relinquish the passive "carer" role and return back to the active "sexual" role in the management of chronic diseases

Table 1. Sexual disorders and common manifestations

\begin{tabular}{lll}
\hline Sexual & \multicolumn{1}{c}{ Disorders } & \multicolumn{1}{c}{ Common manifestation } \\
\hline Pain & Vulvodynia & Pain experienced in the vulva and vagina \\
& Vaginismus & Painful spasm of vaginal muscles that may prevent or occur on penetration \\
& Dyspareunia & Painful sexual intercourse \\
Libido & Libido loss & Reduced sexual desire/interest-mental \\
& Hypersexuality & Excessive sexual urges \\
Arousal & Diminished & Impaired physical signs of arousal disorder \\
& Increased & Persistent genital arousal disorder \\
Orgasm & Anorgasmia & Failure of orgasm \\
& Persistent genital arousal disorder & Excessive orgasms \\
\hline
\end{tabular}




\section{Obstetrics \& Gynecology Science}

Vol. 64, No. 6, 2021

as a form of coping mechanism-catastrophizing or simply denial [13]. This further compounds the psychosexual harm as the woman is left feeling like an invalid with diminished sexual well-being. The exploration and provision of help with reflection on the different aspects of disease should be rendered to both the woman and her partner to minimize the possibility of reinforced poor sexual self-image. Overall, partner engagement needs to be clearly defined in terms of the timing and level of involvement to avoid undermining the management process.

\section{Summary}

Although time-consuming and a little disconcerting for the less-experienced physician, assessing the disease impact on partners, while incorporating support and engagement, in the management of psychosexual harm in women with chronic vulvar dermatoses can be very productive, improving both the response to treatment and the woman's quality of life. The impact of increased virtual consultations and issues related to gender identity with sexual orientation is not fully understood and is open to research.

\section{Conflict of interest}

No potential conflict of interest relevant to this article was reported.

\section{Ethical approval}

This study does not require approval of the Institutional Review Board because no patient data is contained in this article. The study was performed in accordance with the principles of the Declaration of Helsinki.

\section{Patient consent}

Written informed consent and the use of images from patients are not required for the publication.

\section{Funding information}

None.

\section{References}

1. Goldstein AT, Marinoff SC, Christopher K, Srodon M. Prevalence of vulvar lichen sclerosus in a general gynecology practice. J Reprod Med 2005;50:477-80.

2. Micheletti L, Preti M, Bogliatto F, Zanotto-Valentino MC, Ghiringhello B, Massobrio M. Vulval lichen planus in the practice of a vulval clinic. Br J Dermatol 2000;143:134950.

3. O'Keefe RJ, Scurry JP, Dennerstein G, Sfameni S, Brenan J. Audit of 114 non-neoplastic vulvar biopsies. Br J Obstet Gynaecol 1995;102:780-6.

4. Thorstensen KA, Birenbaum DL. Recognition and management of vulvar dermatologic conditions: lichen sclerosus, lichen planus, and lichen simplex chronicus. J Midwifery Womens Health 2012;57:260-75.

5. Sadownik LA, Koert E, Maher C, Smith KB. A qualitative exploration of women's experiences of living with chronic vulvar dermatoses. J Sex Med 2020;17:1740-50.

6 . Fischer GO. The commonest causes of symptomatic vulvar disease: a dermatologist's perspective. Australas J Dermatol 1996;37:12-8.

7. Basson R. Female sexual response: the role of drugs in the management of sexual dysfunction. Obstet Gynecol 2001;98:350-3.

8. Rosenbaum TY, Barnard E, Wilhite M. Psychosexual aspects of vulvar disease. Clin Obstet Gynecol 2015;58:551-5.

9. Basson R. Human sexual response. Handb Clin Neurol 2015;130:11-8.

10. Brotto L, Atallah S, Johnson-Agbakwu C, Rosenbaum T, Abdo C, Byers ES, et al. Psychological and interpersonal dimensions of sexual function and dysfunction. J Sex Med 2016;13:538-71.

11. Lyons KS, Jones KD, Bennett RM, Hiatt SO, Sayer AG. Couple perceptions of fibromyalgia symptoms: the role of communication. Pain 2013;154:2417-26.

12. Dagan M, Sanderman R, Hoff C, Meijerink WJ, Baas $P C$, van Haastert $M$, et al. The interplay between partners' responsiveness and patients' need for emotional 


\section{Obstetrics \& Gynecology Science}

Tolu Adedipe. Partner support in psychosexual aspects

expression in couples coping with cancer. J Behav Med 2014;37:828-38.

13. Brotto LA, Erskine $Y$, Carey M, Ehlen T, Finlayson S, Heywood $\mathrm{M}$, et al. A brief mindfulness-based cognitive behavioral intervention improves sexual functioning versus wait-list control in women treated for gynecologic cancer. Gynecol Oncol 2012;125:320-5.

14. Gardiner L. Psychosexual history-taking in the 21st century: new terminology, new technology and new risks. BJPsych Advances 2019;25:166-76.

15. O'Connell HE, Sanjeevan KV, Hutson JM. Anatomy of the clitoris. J Urol 2005;174:1189-95. 